PAPER

Non-dipole recollision-gated double ionization and observable effects

To cite this article: A Emmanouilidou et al 2017 J. Phys. B: At. Mol. Opt. Phys. 50225602

View the article online for updates and enhancements.
Related content

Frustrated double and single ionization in a two-electron triatomic molecule $\mathrm{H}+3$ A Chen, C Lazarou, $\mathrm{H}$ Price et al.

Non-adiabatic imprints on the electron wave packet in strong field ionization with circular polarization

C Hofmann, T Zimmermann, A Zielinski et al.

Universal time delay in the recollision impact ionization pathway of strong-field nonsequential double ionization Qianguang Li, Yueming Zhou and Peixiang Lu 


\title{
Non-dipole recollision-gated double ionization and observable effects
}

\author{
A Emmanouilidou ${ }^{1}(1)$, T Meltzer $^{1}$ and P B Corkum ${ }^{2}$ \\ ${ }^{1}$ Department of Physics and Astronomy, University College London, Gower Street, London WC1E 6BT, \\ United Kingdom \\ ${ }^{2}$ Joint Laboratory for Attosecond Science, University of Ottawa and National Research Council, 100 \\ Sussex Drive, Ottawa, Ontario, K1A 0R6, Canada
}

E-mail: a.emmanouilidou@ucl.ac.uk

Received 13 June 2017, revised 11 September 2017

Accepted for publication 4 October 2017

Published 31 October 2017

\begin{abstract}
Using a three-dimensional semiclassical model, we study double ionization for strongly driven He fully accounting for magnetic field effects during the propagation in time. For linearly and slightly elliptically polarized laser fields, we show that recollisions and the magnetic field combined act as a gate. This gate favors more transverse-with respect to the electric fieldinitial momenta of the tunneling electron that are opposite to the propagation direction of the laser field. In the absence of non-dipole effects, the transverse initial momentum is symmetric with respect to zero. We find that this asymmetry in the transverse initial momentum gives rise to an asymmetry in a double ionization observable.
\end{abstract}

Keywords: non-sequential double ionization, non-dipole approximation, non-dipole recollision-gating

(Some figures may appear in colour only in the online journal)

\section{Introduction}

Non-sequential double ionization (NSDI) in driven two-electron atoms is a prototype process for exploring the electron-electron interaction in systems driven by intense laser fields. As such, it has attracted a lot of interest $[1,2]$. Most theoretical studies on NSDI are formulated in the framework of the dipole approximation where magnetic field effects are neglected [3]. However, in the general case that the vector potential $\mathbf{A}$ depends on both space and time, an electron experiences a Lorentz force whose magnetic field component is given by $\mathbf{F}_{\mathbf{B}}=q \mathbf{v} \times \mathbf{B}$. We work in the non-relativistic limit. In this limit, magnetic field effects are expected to arise when the amplitude of the electron motion due to $\mathbf{F}_{\mathbf{B}}$ becomes 1 a.u., i.e. $\beta_{0} \approx U_{\mathrm{p}} /(2 \omega c) \approx 1$ a.u. $[4,5]$. $U_{\mathrm{p}}$ is the ponderomotive energy. Non-dipole effects were previously addressed in theoretical studies of the observed ionization of $\mathrm{Ne}^{n+}(n \leqslant 8)$ in ultra-strong fields [6], of non-sequential double ionization [7], of stabilization [8] and of high-order harmonic generation [9-11] as well as in experimental studies $[12,13]$. In recent studies in single ionization (SI), non-dipole effects of the electron momentum distribution along the propagation direction of the laser field were addressed in experimental [14] and theoretical studies [15-19].
In this work, we show that in double ionization the magnetic field in conjunction with the recollision act as a gate. This gate selects a subset of the initial tunneling-electron momenta along the propagation direction of the laser field. Only this subset leads to double ionization. We show this gating in the context of He when driven by an $800 \mathrm{~nm}$ laser field at intensities $1.3 \times 10^{15} \mathrm{~W} \mathrm{~cm}^{-2}, 2 \times 10^{15} \mathrm{~W} \mathrm{~cm}^{-2}$ and $3.8 \times 10^{15} \mathrm{~W} \mathrm{~cm}^{-2}$. This gating is particularly pronounced at the smaller intensities of $1.3 \times 10^{15} \mathrm{~W} \mathrm{~cm}^{-2}$ and $2 \times 10^{15} \mathrm{~W} \mathrm{~cm}^{-2}$ with $\beta_{0}$ equal to 0.18 a.u. and 0.28 a.u., respectively. Therefore it is important at intensities smaller than the intensities satisfying the criterion for the onset of magnetic field effects $\beta_{0} \approx 1$ a.u. [4, 5]. The propagation direction of the laser field is the same as the direction of the $\mathbf{F}_{\mathbf{B}}$ force (to first order). In the current formulation, the change in momentum due to the $\mathbf{F}_{\mathbf{B}}$ force is along the $+y$-axis. The tunneling electron is the electron that initially tunnels in the field-lowered Coulomb potential. When non-dipole effects are fully accounted for during the propagation in time, we show that the y-component of the initial momentum of the tunneling-electron is mostly negative for events leading to double ionization. In the dipole approximation, the initial momentum of the tunneling-electron that is transverse to the direction of the electric field is symmetric with respect to zero. 
The term non-dipole recollision-gated ionization is adopted to describe ionization resulting from an asymmetric distribution of the transverse tunneling-electron initial momentum due to the combined effect of the recollision and the magnetic field. Nondipole recollision-gated ionization is a general phenomenon. We find that it underlies double electron escape in atoms driven by linearly and slightly elliptically polarized laser fields.

Moreover, we show that non-dipole recollision-gated ionization results in an asymmetry in a double ionization observable. Let $\phi \in\left[0^{\circ}, 180^{\circ}\right]$ denote the angle of the final $(t \rightarrow \infty)$ momentum of each escaping electron with respect to the propagation axis of the laser field. The observable in question is $P_{\text {asym }}^{\mathrm{DI}}(\phi)=P^{\mathrm{DI}}(\phi)-P^{\mathrm{DI}}\left(180^{\circ}-\phi\right)$, where $P^{\mathrm{DI}}(\phi)$ is the probability of either one of the two electrons to escape with an angle $\phi . P^{\mathrm{DI}}(\phi)$ and $P_{\text {asym }}^{\mathrm{DI}}(\phi)$ are accessible by kinematically complete experiments. In the dipole approximation, $P_{\text {asym }}^{\mathrm{DI}}(\phi)=0$. When non-dipole effects are accounted for, it is shown that $P_{\text {asym }}^{\mathrm{DI}}(\phi)>0$, for $\phi \in\left[0^{\circ}, 90^{\circ}\right]$. This is in accord with the effect of $\mathbf{F}_{\mathbf{B}}$. We also find that $P_{\text {asym }}^{\mathrm{DI}}(\phi)$ has considerable values over a wide interval of $\phi$ at lower intensities. This latter feature is an unexpected one. For the intensities considered the $\mathbf{F}_{\mathbf{B}}$ force has small magnitude that increases with intensity. Thus, one would expect the distribution $P_{\mathrm{asym}}^{\mathrm{DI}}(\phi)$ to be very narrowly peaked around $90^{\circ}$ with values increasing with intensity.

\section{Model}

We study ionization in strongly driven He using a threedimensional (3D) semiclassical model that fully accounts for the magnetic field during time propagation-3D-SMND model. We developed this model in [20] by extending a previously developed 3D semiclassical model in the framework of the dipole approximation-3D-SMD model [21, 22]. The Hamiltonian describing the interaction of the fixed nucleus two-electron atom with the laser field is given by

$$
\begin{aligned}
H= & \frac{\left(\mathbf{p}_{1}+\mathbf{A}\left(y_{1}, t\right)\right)^{2}}{2}+\frac{\left(\mathbf{p}_{2}+\mathbf{A}\left(y_{2}, t\right)\right)^{2}}{2} \\
& -c_{1} \frac{Z}{\left|\mathbf{r}_{1}\right|}-c_{2} \frac{Z}{\left|\mathbf{r}_{2}\right|}+c_{3} \frac{1}{\left|\mathbf{r}_{1}-\mathbf{r}_{2}\right|}
\end{aligned}
$$

A is the vector potential given by

$$
\mathbf{A}(\mathrm{y}, t)=-\frac{E_{0}}{\omega} \mathrm{e}^{-\left(\frac{c t-y}{c \tau}\right)^{2}}(\sin (\omega t-k y) \hat{x}+\chi \cos (\omega t-k y) \hat{z}),
$$

$\omega, k, E_{0}$ are the frequency, wavenumber, and strength of the electric component of the laser field, respectively, and $\chi$ is the ellipticity. $c$ is the velocity of light and $\tau=\mathrm{FWHM} / \sqrt{\ln 4}$ with FWHM the full-width-half-maximum of the laser field. All Coulomb forces are accounted for by setting $c_{1}=c_{2}=c_{3}=1$. The laser fields considered in the current work are either linearly polarized, $\chi=0$, or have a small ellipticity of $\chi=0.05$. For A given by equation (2), $\mathbf{E}$ and $\mathbf{B}$ are along the $x$ - and $z$-axis, respectively, with small components along the $z$ - and $x$-axis, respectively, for laser fields with $\chi=0.05$. The propagation direction of the laser field and the direction of $\mathbf{F}_{\mathbf{B}}$ are mainly along the $y$-axis. Unless otherwise stated, all Coulomb forces as well as the electric and the magnetic field are fully accounted for during time propagation. To switch off a Coulomb interaction, the appropriate constant is set equal to zero. For example, to switch off the interaction of electron 1 with the nucleus, $c_{1}$ is set equal to zero. Moreover, we address the Coulomb singularity by using regularized coordinates [23] which were also employed in the 3D-SMD model [21, 22].

The initial state in the 3D-SMND model entails one electron tunneling through the field-lowered Coulomb potential. The electron tunnels with a non-relativistic quantum tunneling rate given by the Ammosov-Delone-Krainov (ADK) formula [24, 25]. A non-relativistic ADK rate results in this Gaussian distribution being centered around zero. In [26] non-dipole effects were accounted for in the ADK rate. It was shown that the most probable initial transverse momentum ranges from $0.33 I_{p} / c$ to almost zero with increasing $E_{0} /\left(2 I_{p}\right)^{3 / 2}$, with $I_{p}$ the ionization energy of the tunneling electron. In this work, the smallest intensity considered is $7 \times 10^{14} \mathrm{~W} \mathrm{~cm}^{-2}$ for He. At this intensity, if non-dipole effects are accounted for in the ADK rate, the transverse momentum of the tunneling electron is centered around 0.12 $I_{p}^{\mathrm{He}} / c$ for $\mathrm{He}$ which is $7.9 \times 10^{-4}$ a.u. $\left(I_{p}^{\mathrm{He}}=0.904\right.$ a.u. $)$. In what follows, we neglect this very small asymmetry and describe the distribution of the initial transverse momentum by a Gaussian distribution centered around zero [24, 25]. We do so in order to clearly illustrate an important phenomenon, i.e. non-dipole recollision-gated ionization. In addition, we set the initial momentum along the direction of the electric field equal to zero. The remaining electron is initially described by a microcanonical distribution [27]. We denote the tunneling and bound electrons by electrons 1 and 2, respectively. The 3D-SMND model is described in more detail in [20].

\section{Non-dipole recollision-gated ionization}

In our calculations we consider $\mathrm{He}$ when driven by an $800 \mathrm{~nm}, 12 \mathrm{fs}$ FWHM laser field that is linearly polarized at intensities $1.3 \times 10^{15} \mathrm{~W} \mathrm{~cm}^{-2}, 2 \times 10^{15} \mathrm{~W} \mathrm{~cm}^{-2}$ and $3.8 \times 10^{15} \mathrm{~W} \mathrm{~cm}^{-2}$ and that is slightly elliptically polarized with $\chi=0.05$ at $2 \times 10^{15} \mathrm{~W} \mathrm{~cm}^{-2}$. At these intensities the ponderomotive energy $U_{\mathrm{p}}=E_{0}^{2} /\left(4 \omega^{2}\right)$ is 2.86 a.u., 4.39 a.u. and 8.35 a.u., respectively. Thus, the maximum energy of electron $1,3.17 U_{\mathrm{p}}$, is above the energy needed to ionize $\mathrm{He}^{+}$.

\subsection{Magnetic field asymmetry in $P_{i}^{\mathrm{DI}}(\phi)$}

First, we show that the magnetic field causes an asymmetry in the double ionization probability of electron $\mathrm{i}$ to ionize with an angle $\phi$, which is denoted by $P_{i}^{\mathrm{DI}}(\phi)$ with $i=1,2$ for electrons 1 and 2. $P^{\mathrm{SI}}(\phi)$ is the corresponding probability in SI. $\phi$ is the angle of the final momentum of electron $i$ with respect to the propagation axis of the laser field, i.e. 

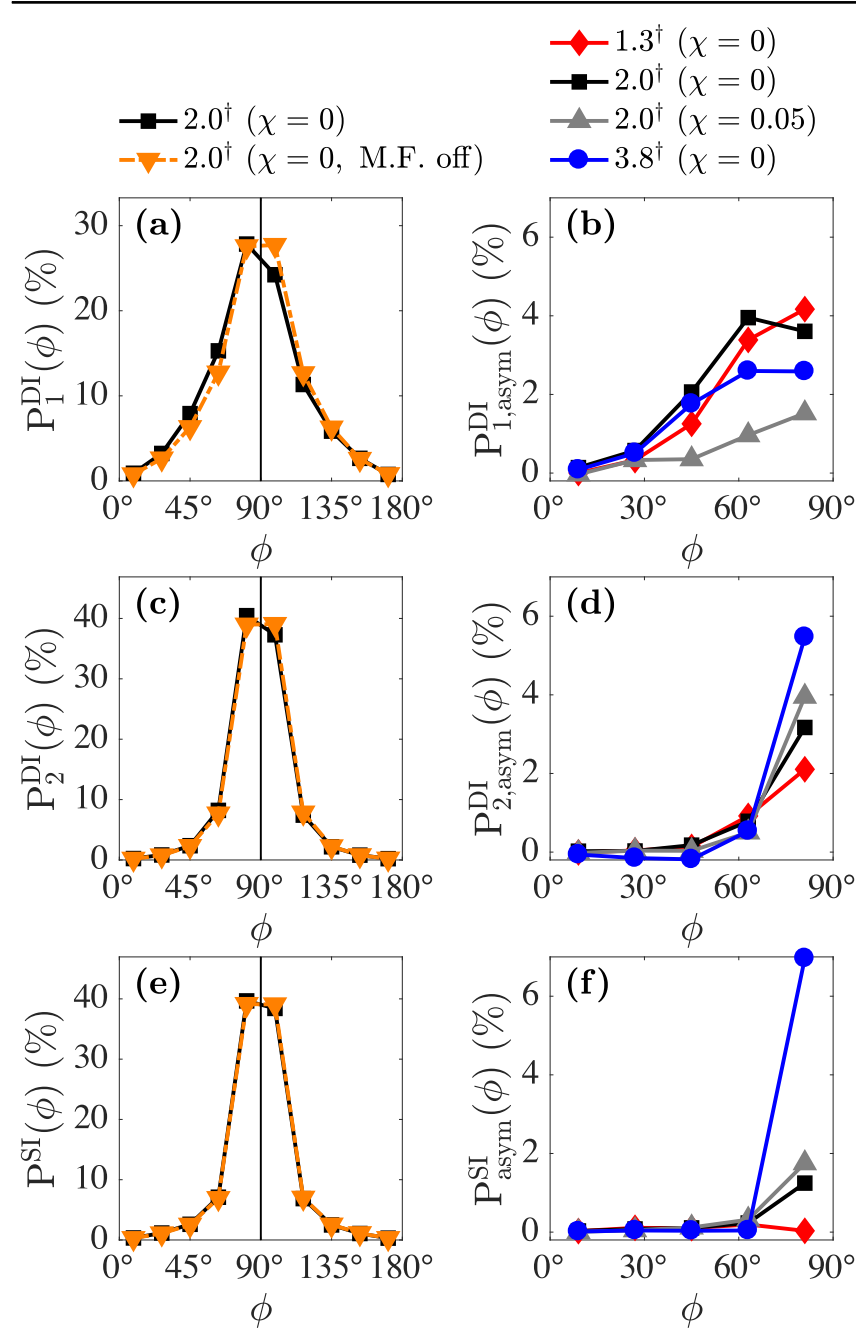

Figure 1. (a) $P_{1}^{\mathrm{DI}}(\phi)$ of the tunneling electron, (c) $P_{2}^{\mathrm{DI}}(\phi)$ of the initially bound electron and (e) $P^{\mathrm{SI}}(\phi)$ in single ionization are plotted as a function of $\phi$ at $2 \times 10^{15} \mathrm{~W} \mathrm{~cm}^{-2}$, with the magnetic field switched-on and off. (b) $P_{1, \text { asym }}^{\mathrm{DI}}(\phi)$ of the tunneling electron, (d) $P_{2, \text { asym }}^{\mathrm{DI}}(\phi)$ of the initially bound electron and (f) $P_{\text {asym }}^{\mathrm{SI}}(\phi)$ in single ionization are plotted as a function of $\phi$ at three intensities with $\chi=0$ and at one intensity with $\chi=0.05$. $\phi$ is binned in intervals of $18^{\circ}$ and $\dagger$ denotes a multiplication factor of $10^{15} \mathrm{Wcm}^{-2}$.

$\cos \phi=p^{i} \cdot \hat{y} /\left|p^{i}\right|$. The y-component of the electron momentum is parallel to the propagation direction of the laser field and to $\mathbf{F}_{\mathbf{B}}$.

To show this asymmetry, we plot $P_{1}^{\mathrm{DI}}(\phi)$ of the tunnel electron and $P_{2}^{\mathrm{DI}}(\phi)$ of the initially bound electron in figures 1(a) and (c), respectively, while we plot $P^{\mathrm{SI}}(\phi)$ in figure $1(\mathrm{e})$. These plots are at an intensity of $2 \times 10^{15} \mathrm{~W} \mathrm{~cm}^{-2}$ with the magnetic field switched-on and off. When the magnetic field is switched-on, we find that all probability distributions are asymmetric with respect to $\phi=90^{\circ}$. This asymmetry is due to the magnetic field. Indeed, when the magnetic field is switchedoff all distributions are shown to be symmetric with respect to $\phi=90^{\circ}$. The latter is expected, since there is no preferred direction of electron escape on the plane that is perpendicular to the $x$-axis (polarization direction). Moreover, with the magnetic field switched-on, we find that $P_{i}^{\mathrm{DI}}(\phi)>P_{i}^{\mathrm{DI}}\left(180^{\circ}-\phi\right)$ and
$P^{\mathrm{SI}}(\phi)>P^{\mathrm{SI}}\left(180^{\circ}-\phi\right)$ for $\phi \in\left[0^{\circ}, 90^{\circ}\right]$. Equivalently $P_{i, \text { asym }}^{\mathrm{DI}}(\phi)=P_{i}^{\mathrm{DI}}(\phi)-P_{i}^{\mathrm{DI}}\left(180^{\circ}-\phi\right)>0$ and $P_{\mathrm{asym}}^{\mathrm{SI}}(\phi)=$ $P^{\mathrm{SI}}(\phi)-P^{\mathrm{SI}}\left(180^{\circ}-\phi\right)>0$ for $\phi \in\left[0^{\circ}, 90^{\circ}\right]$. This is consistent with the gain of momentum due to the $\mathbf{F}_{\mathbf{B}}$ force being along the $+y$-axis. That is, an electron is more likely to ionize with a positive rather than a negative y-component of the final momentum.

The asymmetry with respect to $\phi=90^{\circ}$ is better illustrated in figure 1 . We plot $P_{i \text {,asym }}^{\mathrm{DI}}(\phi)$ and $P_{\mathrm{asym}}^{\mathrm{SI}}(\phi)$ as a function of $\phi$ at $1.3 \times 10^{15} \mathrm{~W} \mathrm{~cm}^{-2}, 2 \times 10^{15} \mathrm{~W} \mathrm{~cm}^{-2}$ and $3.8 \times 10^{15} \mathrm{~W} \mathrm{~cm}^{-2}$ and at $2 \times 10^{15} \mathrm{~W} \mathrm{~cm}^{-2}$ with $\chi=0.05$. Starting with SI, $P_{\text {asym }}^{\text {SI }}(\phi)$ is almost zero at $1.3 \times 10^{15}$ $\mathrm{W} \mathrm{cm}{ }^{-2}$. At the higher intensity of $3.8 \times 10^{15} \mathrm{~W} \mathrm{~cm}^{-2}$, $P_{\text {asym }}^{\mathrm{SI}}(\phi)$ is sharply centered around $90^{\circ}$ reaching roughly $7 \%$, see figure 1(f). These features of $P^{\mathrm{SI}}(\phi)$ are in accord with the effect of the $\mathbf{F}_{\mathbf{B}}$ force. $\mathbf{F}_{\mathbf{B}}$ is small for the intensities considered. Therefore, $\mathbf{F}_{\mathbf{B}}$ has an observable effect mostly when the y-component of the electron momentum is small as well, i.e. for an angle of escape $\phi=90^{\circ}$. In addition, $\left|\mathbf{F}_{\mathbf{B}}\right|$ is three times larger for the higher intensity compared to the smaller one. As a result $P_{\mathrm{asym}}^{\mathrm{SI}}(\phi)$ has larger values at higher intensities.

In double ionization, we plot $P_{2 \text {, asym }}^{\mathrm{DI}}(\phi)$ of the initially bound electron in figure $1(\mathrm{~d})$. It is shown that $P_{2 \text {, asym }}^{\mathrm{DI}}(\phi)$ resembles mostly $P_{\text {asym }}^{\mathrm{SI}}(\phi)$ rather than $P_{1 \text {,asym }}^{\mathrm{DI}}(\phi)$ in figure 1(b). Indeed, $P_{2 \text {, asym }}^{\mathrm{DI}}(\phi)$ has larger values for higher intensities, as is the case for $P_{\text {asym }}^{\mathrm{SI}}(\phi)$, reaching roughly $5.5 \%$ at $3.8 \times 10^{15} \mathrm{~W} \mathrm{~cm}^{-2}$. We also find that the distribution $P_{1, \text { asym }}^{\mathrm{DI}}(\phi)$ of the tunneling electron has different features from $P_{\text {asym }}^{\mathrm{SI}}(\phi)$, compare figure 1(b) with figure $1(\mathrm{f})$. We find that $P_{1, \text { asym }}^{\mathrm{DI}}(\phi)$ is much wider than $P_{\text {asym }}^{\mathrm{SI}}(\phi)$. Also, for $\phi \in\left[45^{\circ}, 90^{\circ}\right], P_{1, \text { asym }}^{\mathrm{DI}}(\phi)$ has higher values up to $2 \times 10^{15}$ $\mathrm{W} \mathrm{cm}{ }^{-2}$ compared to $3.8 \times 10^{15} \mathrm{~W} \mathrm{~cm}^{-2}$ with $4 \%$ and $2.5 \%$, respectively. We have shown in [20] that strong recollisions [29] prevail for strongly driven $\mathrm{He}$ at $800 \mathrm{~nm}$ at intensities of $1.3 \times 10^{15} \mathrm{~W} \mathrm{~cm}^{-2}$ and $2 \times 10^{15} \mathrm{~W} \mathrm{~cm}^{-2}$, while soft ones prevail at $3.8 \times 10^{15} \mathrm{~W} \mathrm{~cm}^{-2}$. It then follows that $P_{1, \text { asym }}^{\mathrm{DI}}(\phi)$ has higher values for strong recollisions. This is also supported by the small values of $P_{1, \text { asym }}^{\mathrm{DI}}(\phi)$ at $2 \times 10^{15} \mathrm{~W} \mathrm{~cm}^{-2}$ for a laser pulse with a small ellipticity of $\chi=0.05$, see figure 1(b). We find (not shown) that the recollisions are soft at $2 \times 10^{15} \mathrm{~W} \mathrm{~cm}^{-2}$ for a laser pulse with $\chi=0.05$. The times of recollision correspond roughly to zeros of the laser field for strong recollisions and extrema of the laser field for soft ones [20, 28]. Moreover, the transfer of energy, compared to $U_{\mathrm{p}}$, from electron 1 to 2 is larger for a strong recollision and smaller for a soft one [20, 28]. Later in the paper, we explain in detail why the width and the values of $P_{1 \text {, asym }}^{\mathrm{DI}}(\phi)$ are large at $2 \times 10^{15} \mathrm{~W} \mathrm{~cm}^{-2}$, smaller at $3.8 \times 10^{15} \mathrm{~W} \mathrm{~cm}^{-2}$ and even smaller at $2 \times 10^{15} \mathrm{~W} \mathrm{~cm}^{-2}$ for a laser pulse with $\chi=0.05$.

Experimentally electron 1 can not be distinguished from electron 2 . Therefore, the probability distributions of electrons 1 and 2 to escape with an angle $\phi, P_{1}^{\mathrm{DI}}(\phi)$ (figure 1(a)) and $P_{2}^{\mathrm{DI}}(\phi)$ (figure $1(\mathrm{c})$ ), respectively, are not experimentally accessible. However, in a kinematically complete experiment, 


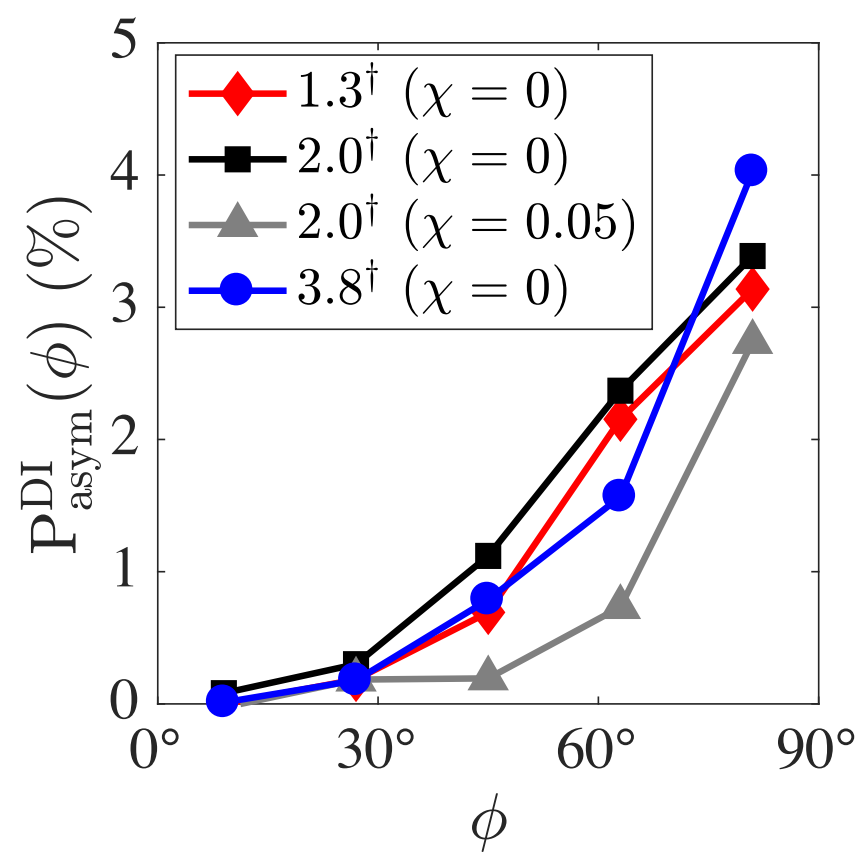

Figure 2. In double ionization, $P_{\mathrm{asym}}^{\mathrm{DI}}(\phi)$ for the two escaping electrons is plotted as a function of $\phi$. Three intensities are considered and $\phi$ is binned in intervals of $18^{\circ}$. $\dagger$ Denotes a multiplication factor of $10^{15} \mathrm{~W} \mathrm{~cm}{ }^{-2}$.

for each doubly ionized event, the angle $\phi$ of each ionizing electron can be measured. Then, the probability distribution for any one of the two electrons to ionize with an angle $\phi$, $P^{\mathrm{DI}}(\phi)$, can be obtained, for $\phi \in\left[0^{\circ}, 180^{\circ}\right]$. We compute and plot the distribution $P_{\text {asym }}^{\mathrm{DI}}(\phi)$ as a function of $\phi$ in figure 2 . $P_{\text {asym }}^{\mathrm{DI}}(\phi)$ is found to have significant values at smaller intensities over the same wide range of $\phi$ as $P_{1 \text {,asym }}^{\mathrm{DI}}(\phi)$ does. However, $P_{\text {asym }}^{\mathrm{DI}}(\phi)<P_{1 \text {, asym }}^{\mathrm{DI}}(\phi)$ at the smaller intensities. Moreover, at $3.8 \times 10^{15} \mathrm{~W} \mathrm{~cm}^{-2}$ and at $2 \times 10^{15} \mathrm{~W} \mathrm{~cm}^{-2}$ with $\chi=0.05, P_{\mathrm{asym}}^{\mathrm{DI}}(\phi)>P_{1, \text { asym }}^{\mathrm{DI}}(\phi)$ at $\phi=81^{\circ}$ but has non zero values for a wider range of $\phi$ compared to $P_{2 \text {, asym }}^{\text {DI }}(\phi)$. These features are expected since $P_{\text {asym }}^{\mathrm{DI}}(\phi)$ accounts for both the tunneling and the initially bound electron. However, the features of the experimentally accessible $P_{\text {asym }}^{\mathrm{DI}}(\phi)$ still capture the main features of $P_{1, \text { asym }}^{\mathrm{DI}}(\phi)$. In addition, to compare with future experiments, in our computations we would have to account for intensity averaging over the focal volume [30]. Using our current results for intensities from $0.7 \times 10^{15} \mathrm{~W} \mathrm{~cm}^{-2}$ to $3.8 \times 10^{15} \mathrm{~W} \mathrm{~cm}^{-2}$ we can roughly estimate how intensity averaging affects the distribution $P_{\mathrm{asym}}^{\mathrm{DI}}(\phi)$; for a more accurate estimate, results at more intensities than currently considered are required. We find that the resulting distributions $P_{\mathrm{asym}}^{\mathrm{DI}}(\phi)$ at $1.3 \times 10^{15} \mathrm{~W} \mathrm{~cm}^{-2}$ and $2 \times 10^{15} \mathrm{~W} \mathrm{~cm}^{-2}$ do not have as large values as the distributions in figure 2. However, the resulting distributions still have non zero values for a wide range of $\phi$. Moreover, when intensity averaging over the focal volume is considered, the distribution $P_{\mathrm{asym}}^{\mathrm{DI}}(\phi)$ has similar values at $3.8 \times 10^{15}$ $\mathrm{W} \mathrm{cm}{ }^{-2}$ and at $1.3 \times 10^{15} \mathrm{~W} \mathrm{~cm}^{-2}$ and $2 \times 10^{15} \mathrm{~W} \mathrm{~cm}^{-2}$. Therefore, intensities higher than $3.8 \times 10^{15} \mathrm{~W} \mathrm{~cm}^{-2}$ have to be considered experimentally in order to observe the
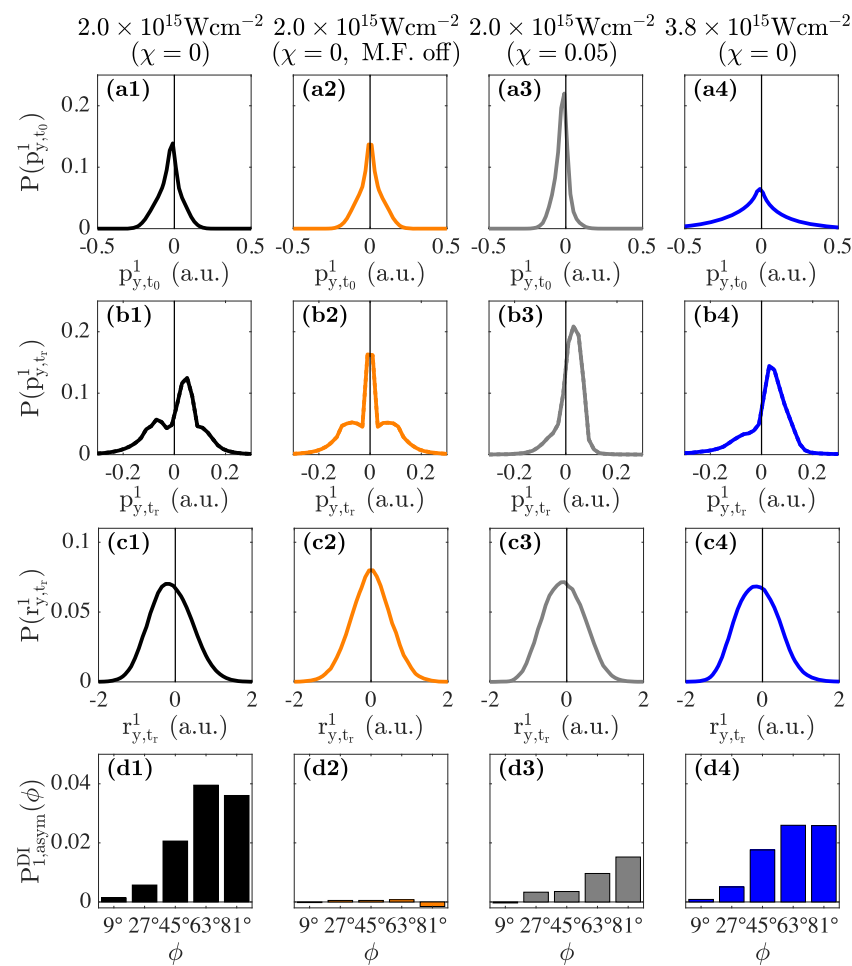

Figure 3. In double ionization, the distributions of the y-component of the electron 1 momentum at two different times are plotted; (a1)(a4) at the time electron 1 tunnel-ionizes (momentum $p_{y, t_{0}}^{1}$ ); (b1)(b4) at the time just before the time of recollision (momentum $p_{y, t_{\mathrm{r}}}^{1}$ ). The distributions of the y-component of the electron 1 position are plotted (c1)-(c4) at the time just before the time of recollision (position $\left.r_{y, t_{\mathrm{r}}}^{1}\right) \cdot P_{1, \text { asym }}^{\mathrm{DI}}(\phi)$ is plotted in panels (d1)-(d4) for better comparison of its features with the features of $P\left(p_{y, t_{\mathrm{r}}}^{1}\right)$. Panels (d1), (d3) and (d4) are the same as the plots in figure 1(b).

transition shown in figure 2 where the distribution $P_{\text {asym }}^{\mathrm{DI}}(\phi)$ at $3.8 \times 10^{15} \mathrm{~W} \mathrm{~cm}^{-2}$ has smaller values over a less wide range of $\phi$ compared to its values at $1.3 \times 10^{15} \mathrm{~W} \mathrm{~cm}^{-2}$ and $2 \times 10^{15} \mathrm{~W} \mathrm{~cm}^{-2}$.

\subsection{Asymmetric transverse electron 1 momentum at the tunnel time}

In the following sections we discuss the mechanism responsible for the features of $P_{1, \text { asym }}^{\mathrm{DI}}(\phi)$ and therefore for the features of the observable $P_{\mathrm{asym}}^{\mathrm{DI}}(\phi)$. We find that this mechanism is a signature of recollision exclusive to non-dipole effects. We find that the magnetic field and the recollision act together as a gate that selects only a subset of transverse initial momenta of the tunneling electron that lead to double ionization. For linearly polarized light, this gating is illustrated in figures $3(\mathrm{a} 1)$ and (a2) at an intensity of $2 \times 10^{15} \mathrm{~W} \mathrm{~cm}^{-2}$ with the magnetic field switched-on and off, respectively. We plot the probability distribution $P\left(p_{y, t_{0}}^{1}\right)$ of electron 1 to tunnel-ionize with a y-component of the initial momentum equal to $p_{y, t_{0}}^{1}$. We find that $P\left(p_{y, t_{0}}^{1}\right)$ is asymmetric when the magnetic field is switched-on. Specifically, it is more likely for electron 1 to tunnel-ionize with a negative rather than a positive $y$-component of the initial momentum. In addition, 
$P\left(p_{y, t_{0}}^{1}\right)$ peaks around small negative values of the momentum of electron 1. Instead, $P\left(p_{y, t_{0}}^{1}\right)$ is symmetric when the magnetic field is switched-off. We also find that $P\left(p_{z, t_{0}}^{1}\right)$ is symmetric around zero (not shown) when the magnetic field is switched-on and off. This is expected since there is no force acting along the $z$-axis due to the laser field.

We find that this asymmetry in $P\left(p_{y, t_{0}}^{1}\right)$ persists at a higher intensity of $3.8 \times 10^{15} \mathrm{~W} \mathrm{~cm}^{-2}$ for linearly polarized light, see figure $3(\mathrm{a} 4)$. We find that an asymmetry in $P\left(p_{y, t_{0}}^{1}\right)$ is also present at $2 \times 10^{15} \mathrm{~W} \mathrm{~cm}^{-2}$ for a laser pulse with a small ellipticity of $\chi=0.05$, see figure $3(\mathrm{a} 3)$. In contrast, for all the above cases, we find that the initially bound electron has a symmetric distribution $P\left(p_{y, t_{0}}^{2}\right)$ at the time electron 1 tunnel-ionizes and just before the time of recollision. In our analysis, this time is identified for each double ionizing trajectory as the time that the electron-electron potential energy $1 /\left|\mathbf{r}_{1}-\mathbf{r}_{2}\right|$ as a function of time is maximum. Moreover, in SI we find that the escaping electron has a symmetric distribution $P\left(p_{y, t_{0}}\right)$ at the time this electron tunnel-ionizes.

\subsection{Asymmetric transverse electron 1 momentum shortly before recollision}

In SI, to understand the features of $P_{\text {asym }}^{\mathrm{SI}}(\phi)$ one must obtain the distribution $P\left(p_{y, t_{0}}^{1}\right)$ at the time electron 1 tunnel-ionizes. We therefore compute the $\mathrm{y}$-component of the escaping electron's momentum both with all Coulomb forces switchedoff and with all Coulomb forces accounted for. In both cases we start the time propagation from the instant electron 1 tunnel-ionizes. We have shown in [20] that the average $\mathrm{y}$-component of the electron 1 momentum is roughly the same for both cases. At the time electron 1 tunnel-ionizes, we find that the y-component of the transverse momentum of electron 1 is roughly symmetric around zero. This initial momentum distribution combined with the $\mathbf{F}_{\mathbf{B}}$ force give rise to the electron ionizing mostly with $\phi$ slightly less than $90^{\circ}$ or equivalently give rise to a sharply peaked distribution $P_{\text {asym }}^{\mathrm{SI}}(\phi)$ (figure 1(f)).

In double ionization, to understand the features of $P_{1, \text { asym }}^{\text {DI }}(\phi)$, first, we must obtain the distribution $P\left(p_{y, t_{\mathrm{r}}}^{1}\right)$ of the $\mathrm{y}$-component of the electron 1 momentum shortly before the time of recollision. Then, we must find the effect of the recollision itself on the distribution $P\left(p_{y, t_{\mathrm{r}}}^{1}\right)$. The validity of these two steps is supported by the following computations. We propagate the y-component of the momentum of electrons 1 and 2 from the time electron 1 tunnel-ionizes up to the time of recollision. We do so using the full 3D-SMND model with all Coulomb forces accounted for. Next, using as initial conditions the momenta of electrons 1 and 2 shortly after the time of recollision, we propagate from the time of recollision onwards with all Coulomb forces and the magnetic field switched-off. The final average y-component of the momentum of electron 1 is roughly equal in both cases and the same holds for electron 2 [20]. Therefore, the decisive time in double ionization is the time of recollision.
Given the above, we first compute $P\left(p_{y, t_{\mathrm{r}}}^{1}\right)$ just before the time of recollision. This is done by extracting from the full 3D-SMND model the distribution of the y-component of the electron 1 momentum at a time just before the recollision, for instance at $t_{\mathrm{bef}}=t_{\mathrm{r}}-1 / 50 T$. $T$ is the period of the laser field. We choose $t_{\text {bef }}$ such as to avoid the sharp change of the momenta which occurs at $t_{\mathrm{r}}$, see [20]. At all intensities considered, we find that shortly before the time of recollision a positive over a negative y-component of electron 1 momentum is favored. This is shown at intensities of $2 \times 10^{15}$ $\mathrm{W} \mathrm{cm}{ }^{-2}$ and $3.8 \times 10^{15} \mathrm{~W} \mathrm{~cm}^{-2}$ for linearly polarized light in figures 3(b1) and (b4), respectively, and at $2 \times 10^{15}$ $\mathrm{W} \mathrm{cm}{ }^{-2}$ for elliptically polarized light with $\chi=0.05$ in figure 3(b3). In contrast, when the magnetic field is switchedoff the distribution $P\left(p_{y, t_{\mathrm{r}}}^{1}\right)$ is symmetric with respect to zero as illustrated for an intensity of $2 \times 10^{15} \mathrm{~W} \mathrm{~cm}^{-2}$ in figure 3(b2).

We have now established that the shift towards negative momenta of $P\left(p_{y, t_{0}}^{1}\right)$ at the time electron 1 tunnel-ionizes maps to a shift towards positive momenta of $P\left(p_{y, t_{\mathrm{r}}}^{1}\right)$ just before the time of recollision. The width is another interesting feature of the distribution $P\left(p_{y, t_{0}}^{1}\right)$ and, by extension, of the distribution $P\left(p_{y, t_{\mathrm{r}}}^{1}\right)$. From figures 3(b1)-(b4), we find that the width of $P\left(p_{y, t_{\mathrm{r}}}^{1}\right)$ at $2 \times 10^{15} \mathrm{~W} \mathrm{~cm}^{-2}$ with linearly polarized light is the largest one, while the width of $P\left(p_{y, t_{\mathrm{r}}}^{1}\right)$ at $2 \times 10^{15}$ $\mathrm{W} \mathrm{cm}{ }^{-2}$ with $\chi=0.05$ is the smallest one. Moreover, the width of $P\left(p_{y, t_{0}}^{1}\right)$ is comparable with the width of $P\left(p_{y, t_{\mathrm{r}}}^{1}\right)$ for both the linear and the elliptical laser field at $2 \times 10^{15}$ $\mathrm{W} \mathrm{cm}{ }^{-2}$. However, the width of $P\left(p_{y, t_{0}}^{1}\right)$ is larger than the width of $P\left(p_{y, t_{\mathrm{r}}}^{1}\right)$ at an intensity of $3.8 \times 10^{15} \mathrm{~W} \mathrm{~cm}^{-2}$.

We find that the widths of $P\left(p_{y, t_{0}}^{1}\right)$ and $P\left(p_{y, t_{\mathrm{r}}}^{1}\right)$ are consistent with Coulomb focusing which mainly refers to multiple returns of electron 1 to the core $[31,32]$. In addition, for the larger intensity of $3.8 \times 10^{15} \mathrm{~W} \mathrm{~cm}^{-2}$, the widths are also consistent with a larger effect of the Coulomb potential of the ion on electron 1 . The latter effect is not due to multiple returns of electron 1 to the core but rather due to electron 1 tunnel-ionizing closer to the nucleus at higher intensities.

In table 1 we show the number of times electron 1 returns to the core before it finally escapes. We find that electron 1 returns more times to the core at $2 \times 10^{15} \mathrm{~W} \mathrm{~cm}^{-2}$. Namely, electron 1 escapes with only one return to the core in $27 \%$ of doubly ionized events. Also, it returns roughly the same number of times at $2 \times 10^{15} \mathrm{~W} \mathrm{~cm}^{-2}$ with $\chi=0.05$ and at $3.8 \times 10^{15} \mathrm{~W} \mathrm{~cm}^{-2}$. In these two latter cases, electron 1 escapes with only one return to the core in more than $50 \%$ of doubly ionized events. We also find (not shown) that the width of the distributions $P\left(p_{y, \mathrm{t}_{0}}^{1}\right)$ and $P\left(p_{y, t_{\mathrm{r}}}^{1}\right)$ increases with increasing number of returns to the core for all laser fields considered. The above features are consistent with Coulomb focusing. Thus, Coulomb focusing explains why the width of $P\left(p_{y, t_{0}}^{1}\right)$ at $2 \times 10^{15} \mathrm{~W} \mathrm{~cm}^{-2}$ is larger than the width of $P\left(\mathrm{p}_{y, t_{0}}^{1}\right)$ at $2 \times 10^{15} \mathrm{~W} \mathrm{~cm}^{-2}$ with $\chi=0.05$.

Moreover, at the larger intensity of $3.8 \times 10^{15} \mathrm{~W} \mathrm{~cm}^{-2}$ electron 1 exits the field-lowered Coulomb barrier closer to 
Table 1. Number of returns to the core of electron 1 for doubly ionized events.

\begin{tabular}{lcccc}
\hline & $\begin{array}{c}2.0^{\mathrm{a}} \\
(\chi=0)\end{array}$ & $\begin{array}{c}2.0^{\mathrm{a}} \\
(\chi=0, \text { M.F. off })\end{array}$ & $\begin{array}{c}2.0^{\mathrm{a}} \\
(\chi=0.05)\end{array}$ & $\begin{array}{c}3.8^{\mathrm{a}} \\
(\chi=0)\end{array}$ \\
\hline 1 Return & $27 \%$ & $24 \%$ & $53 \%$ & $57 \%$ \\
2 Returns & $24 \%$ & $24 \%$ & $10 \%$ & $16 \%$ \\
3 Returns & $27 \%$ & $27 \%$ & $19 \%$ & $14 \%$ \\
$>3$ Returns & $21 \%$ & $25 \%$ & $18 \%$ & $12 \%$ \\
\hline
\end{tabular}

Intensities given in units of $10^{15} \mathrm{~W} \mathrm{~cm}^{-2}$.

Table 2. Average distance from the nucleus of electron 1 at the time electron 1 tunnel-ionizes, $\left\langle r_{t_{0}}^{1}\right\rangle$.

\begin{tabular}{ccccc}
\hline & $\begin{array}{c}2.0^{\mathrm{a}} \\
(\chi=0)\end{array}$ & $\begin{array}{c}2.0^{\mathrm{a}} \\
(\chi=0, \text { M.F. off })\end{array}$ & $\begin{array}{c}2.0^{\mathrm{a}} \\
(\chi=0.05)\end{array}$ & $\begin{array}{c}3.8^{\mathrm{a}} \\
(\chi=0)\end{array}$ \\
\hline$\left\langle r_{t_{0}}^{1}\right\rangle$ (a.u.) & 3.5 & 3.5 & 3.7 & 2.4 \\
\hline
\end{tabular}

Intensities given in units of $10^{15} \mathrm{~W} \mathrm{~cm}^{-2}$.

the nucleus. Indeed, we find that the average distance of electron 1 from the nucleus at the time electron 1 tunnelionizes is 2.4 a.u. at $3.8 \times 10^{15} \mathrm{~W} \mathrm{~cm}^{-2}$ compared to roughly 3.5 a.u. at $2 \times 10^{15} \mathrm{~W} \mathrm{~cm}^{-2}$ with $\chi=0$ and $\chi=0.05$, see table 2. This is consistent with the width of $P\left(p_{y, t_{\mathrm{r}}}^{1}\right)$ being significantly smaller than the width of $P\left(p_{y, t_{0}}^{1}\right)$ at $3.8 \times 10^{15}$ $\mathrm{W} \mathrm{cm}{ }^{-2}$. That is, the Coulomb potential of the ion has a large effect on electron 1 from the time electron 1 tunnel-ionizes onwards. This is not the case for the smaller intensities of $2 \times 10^{15} \mathrm{~W} \mathrm{~cm}^{-2}$ with $\chi=0$ and $\chi=0.05$ where the widths of the distributions $P\left(p_{y, t_{0}}^{1}\right)$ and $P\left(p_{y, t_{\mathrm{r}}}^{1}\right)$ are similar.

\subsection{Glancing angles in recollisions}

We have shown in the previous section that effectively the only force that could result in a change of the momenta of the two electrons between the final time and the time shortly after recollision is due to the electric field. Between the time shortly after recollision takes place and the asymptotic time, the electric field mainly affects the x-component and not the $y$-component of the momentum of electron 1. Moreover, at the smaller intensities considered, in the time interval following recollision, the electric field does not affect significantly the magnitude of the x-component of the electron 1 momentum; this component is mainly determined by the vector potential at the recollision time. Given the above, it is enough to find how the momentum of electron 1 (roughly equal to the $\mathrm{x}$-component) and its $\mathrm{y}$-component change from just before to just after the time of recollision due to the recollision itself. This will allow us to understand the features of the distribution $P_{1 \text {,asym }}^{\text {DI }}(\phi)$ of the final angle $\phi$.

A measure of the strength of a recollision is the angle $\theta$, where $\cos \theta=p_{1, \text { bef }} \cdot p_{1, \text { aft }} /\left(\left|p_{1, \text { bef }}\right|\left|p_{1, \text { aft }}\right|\right)$. That is, $\theta$, is the angle between the momentum of electron 1 just before and just after the time of recollision. The momentum just before the time of recollision is roughly along the $x$-axis (polarization axis). Thus, $\theta$ is the angle of the momentum of electron 1 after the time of recollision with respect to the $x$-axis.
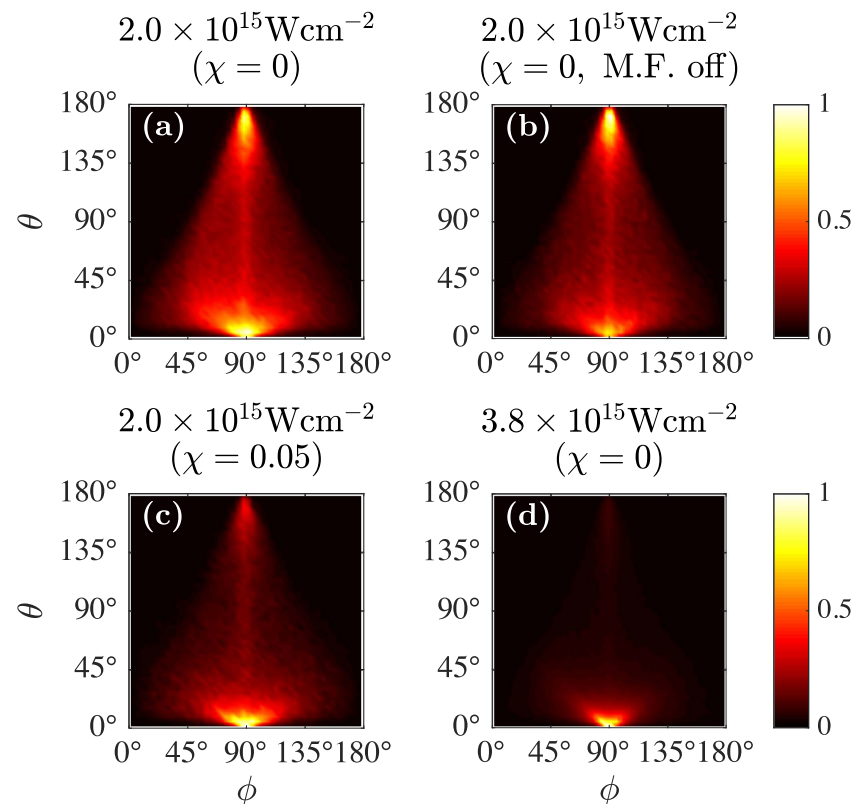

Figure 4. Double differential probability of electron 1 to have a scattering angle $\theta$ and a final angle $\phi$ at (a) $2 \times 10^{15} \mathrm{~W} \mathrm{~cm}^{-2}$, (b) $2 \times 10^{15} \mathrm{~W} \mathrm{~cm}^{-2}$ with the magnetic field switched-off, (c) $2 \times 10^{15}$ $\mathrm{W} \mathrm{cm}{ }^{-2}$ with $\chi=0.05$ and (d) $3.8 \times 10^{15} \mathrm{~W} \mathrm{~cm}^{-2}$.

$\theta=180^{\circ}$ corresponds to a 'head on' collision and complete backscattering. $\theta=0^{\circ}$ corresponds to forward scattering and thus to almost no change due to the recollision.

In figure 4, we show for each laser field considered in this work, what is the probability for electron 1 to escape with a final angle $\phi$ and a scattering angle $\theta$. It is shown that very strong recollisions, i.e. $\theta=180^{\circ}$, take place only when electron 1 escapes with a momentum that has a very small y-component, i.e. $\phi$ is around $90^{\circ}$. However, even when electron 1 escapes with $\phi$ around $90^{\circ}$, it is more likely that a weak recollision takes place, i.e. $\theta=0^{\circ}$, rather than a strong one with $\theta=180^{\circ}$. Moreover, when electron 1 ionizes with momenta that have larger y-components with $\phi \in\left[45^{\circ}, 90^{\circ}\right]$ and $\phi \in\left[90^{\circ}, 135^{\circ}\right]$ the scattering angles $\theta$ are on average smaller than $90^{\circ}$. That is, in most cases, electron 1 ionizes at glancing angles $\theta$ following recollision. Moreover, a comparison of the values of $\theta$ at $2 \times 10^{15} \mathrm{~W} \mathrm{~cm}^{-2}$ and at $3.8 \times 10^{15} \mathrm{~W} \mathrm{~cm}^{-2}$, see figures $4(\mathrm{a})$ and (d), clearly shows that overall the recollision is stronger at the smaller intensity. In addition, a comparison of the values of $\theta$ at $2 \times 10^{15}$ $\mathrm{W} \mathrm{cm}{ }^{-2}$ and at $2 \times 10^{15} \mathrm{~W} \mathrm{~cm}^{-2}$ with $\chi=0.05$, see figures 4(a) and (c), clearly shows that overall the recollision 
is stronger when $\chi=0$. Thus, electron 1 escapes at glancing angles following recollision.

\subsection{Asymmetric transverse electron 1 position shortly before recollision}

We first explain how the y-component of the momentum of electron 1 changes from just before to just after the time of recollision, due to the recollision itself, when the magnetic field is switched-off at $2 \times 10^{15} \mathrm{~W} \mathrm{~cm}^{-2}$. We find that doubly ionized events are equally likely to have positive or negative y-component of the momentum and of the position of electron 1 just before the time of recollision, see figures 3(b2) and (c2). Moreover, we find that the $\mathrm{y}$-component of the final momentum of electron 1 is positive (negative) depending on whether the y-component of the position of electron 1 is negative (positive) just before the time of recollision. The reason is that electron 1 ionizes at glancing angles. The direction of the Coulomb attraction of electron 1 from the nucleus just before the time of recollision determines whether just after the time of recollision, as well as at asymptotically large times, the y-component of the momentum of electron 1 is positive or negative. Indeed, we find that doubly ionized events with $r_{y, t_{\mathrm{r}}}^{1}>0$ give rise to negative values of $P_{1, \text { asym }}^{\mathrm{DI}}$, while doubly ionized events with $r_{y, t_{\mathrm{r}}}^{1}<0$ give rise to positive values of $P_{1, \text { asym }}^{\mathrm{DI}}$ and cancel each other out.

However, when the magnetic field is switched-on at $2 \times 10^{15} \mathrm{~W} \mathrm{~cm}^{-2}$, most (59\%) doubly ionized events have $r_{y, t_{r}}^{1}<0$ just before the time of recollision. This is due to nondipole recollision-gated ionization, since out of these latter events $61 \%$ have both $p_{y, t_{r}}^{1}>0$ just before the time of recollision and $p_{y, t_{0}}^{1}<0$ at the initial time that electron 1 tunnel-ionizes. The shift towards negative values in the transverse y-component of the position of electron 1 just before the time of recollision is shown in figures $3(\mathrm{c} 1)$ and (c3) at $2 \times 10^{15} \mathrm{~W} \mathrm{~cm}^{-2}$ with $\chi=0$ and $\chi=0.05$, respectively, and in (c4) at $3.8 \times 10^{15} \mathrm{~W} \mathrm{~cm}^{-2}$. As for the case when the magnetic field is switched-off, when the magnetic field is switched-on electron 1 ionizes at glancing angles. Therefore, we find that doubly ionized events with $r_{y, t_{r}}^{1}>0$ give rise to negative values of $P_{1 \text {,asym }}^{\mathrm{DI}}$ and doubly ionized events with $r_{y, t_{\mathrm{r}}}^{1}<0$ give rise to positive values of $P_{1, \text { asym }}^{\mathrm{DI}}$. However, the latter events are 59\% of all doubly ionized events and thus overall $P_{1, \text { asym }}^{\mathrm{DI}}$ has positive values.

From the above it also follows that a small width of $P\left(p_{y, t_{\mathrm{r}}}^{1}\right)$ affects only doubly ionized events with small $y$-components of the electron 1 final momenta. On the other hand, a large width of $P\left(p_{y, t_{\mathrm{r}}}^{1}\right)$ just before the time of recollision affects doubly ionized events with y-components of the electron 1 final momenta ranging from small to large. However, doubly ionized events with large y-components of the electron 1 momenta correspond to smaller $\phi$ in $P_{1, \text { asym }}^{\mathrm{DI}}$. Indeed, this is clear in figure 5 when comparing the distribution of the y-component of the final momentum of electron 1 in the smaller $\phi$ of $27^{\circ}$ with the one in the larger $\phi$ of $81^{\circ}$. The large y-components of the momenta of electron
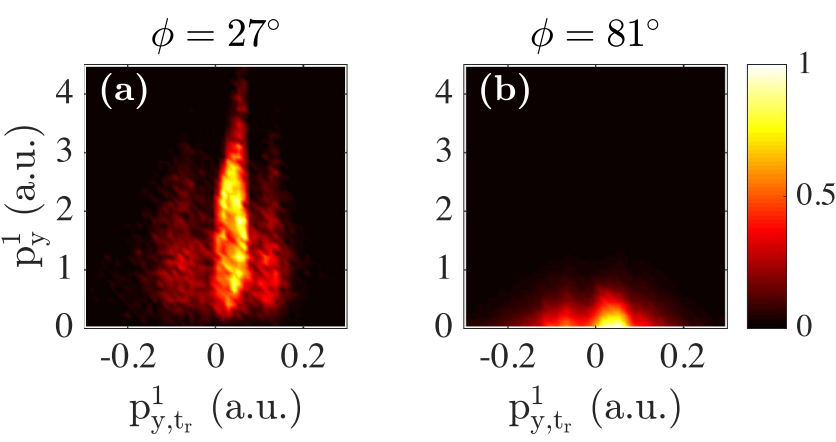

Figure 5. Double differential probability of the tunneling electron to have a y-component of the momentum equal to $p_{y, t_{\mathrm{r}}}^{1}$ at the time of recollision and equal to $p_{y}^{1}$ at the asymptotic time for two different $\phi s$ at $2.0 \times 10^{15} \mathrm{~W} \mathrm{~cm}^{-2} . \phi$ is binned in intervals of $18^{\circ}$.

1 are a result of the recollision. This is the case since for each intensity, $P\left(p_{y, t_{\mathrm{r}}}^{1}\right)$ are similar for all $\phi$ bins, see figure 5 . $P\left(p_{y, t_{r}}^{1}\right)$ is depicted in figures 3(b1), (b3) and (b4) for three different laser fields. Most doubly ionized events just before the time of recollision have positive y-component of the momentum of electron 1 . Thus, the width of $P\left(p_{y, t_{\mathrm{r}}}^{1}\right)$ is also a measure of the shift towards positive y-components of the final momenta of electron 1.

Finally, we compare the width of the distribution of the $\mathrm{x}$-component of the momentum of electron 1 at intensities where strong recollisions prevail with the width at intensities where soft ones do. We find that this width is larger just before and just after the time of recollision as well as at asymptotically large times at intensities where soft recollisions prevail. Indeed, for strong recollisions, the $\mathrm{x}$-component of the momentum of electron 1 is determined mainly by the vector potential at the time of recollision. However, for soft recollisons this is not quite the case since electron 1 only transfers a small amount of its energy to electron 2. In addition, for soft recollisions, the time of recollison has a much broader range of values, see [20].

We now combine the width of the asymmetry of $P\left(p_{y}^{1}\right)$ with the width of the distribution of the x-component of the momentum of electron 1 . It then follows that $P_{1, \text { asym }}^{\mathrm{DI}}(\phi)$ should have higher values over a larger range of $\phi$ at $2 \times 10^{15} \mathrm{~W} \mathrm{~cm}^{-2}$ (stronger recollision) and smaller values at $2 \times 10^{15} \mathrm{~W} \mathrm{~cm}^{-2}$ with $\chi=0.05$ (softer recollision). Indeed, this is the case as shown in figures $3(\mathrm{~d} 1)-(\mathrm{d} 4)$.

\section{Average sum electron momenta in double ionization}

In [20], we have shown that in double ionization the ratio $\left\langle p_{y}^{1}+p_{y}^{2}\right\rangle_{\mathrm{DI}} / 2\left\langle p_{y}\right\rangle_{\mathrm{SI}}$ is maximum and roughly equal to eight at intensities $1.3 \times 10^{15} \mathrm{~W} \mathrm{~cm}^{-2}$ and $2 \times 10^{15} \mathrm{~W} \mathrm{~cm}^{-2}$, see figure $6 .\left\langle p_{y}^{1}+p_{y}^{2}\right\rangle$ is the average sum of the two-electron momenta along the propagation direction of the laser field, while $\left\langle p_{y}\right\rangle_{\mathrm{SI}}$ is the corresponding average electron momentum in SI. In figure $6(\mathrm{a}),\left\langle p_{y}^{1}+p_{y}^{2}\right\rangle_{\mathrm{DI}} / 2\left\langle p_{y}\right\rangle_{\mathrm{SI}}$ is shown to 

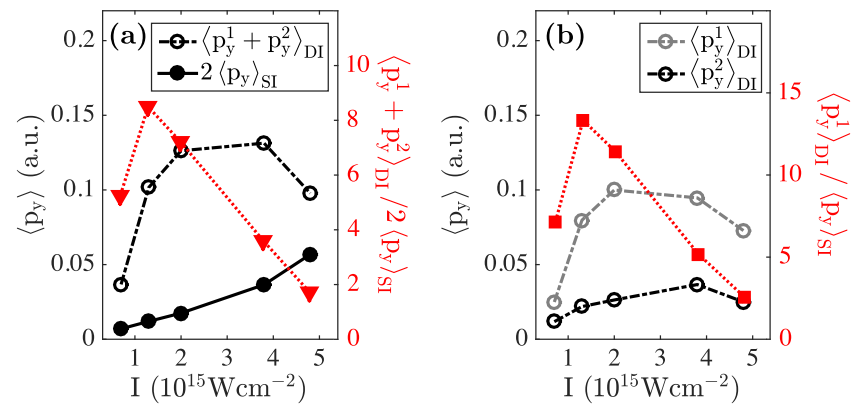

Figure 6. (a) The average sum of the two-electron momenta $\left\langle p_{y}^{1}+p_{y}^{2}\right\rangle_{\mathrm{DI}}$ in double ionization (black dotted-dashed line with open circles), twice the average electron momentum $\left\langle p_{y}\right\rangle_{\mathrm{SI}}$ in single ionization (black solid line with circles) and the ratio $\left\langle p_{y}^{1}+p_{y}^{2}\right\rangle_{\mathrm{DI}} /\left\langle p_{y}\right\rangle_{\mathrm{SI}}$ (red dotted line with triangles) as a function of the intensity of the laser field. (b) The average momentum of the tunneling electron $\left\langle p_{y}^{1}\right\rangle_{\mathrm{DI}}$ (gray dotted-dashed line with open circles) and the bound electron $\left\langle p_{y}^{2}\right\rangle_{\mathrm{DI}}$ (black dotted-dashed line with circles) in double ionization and the ratio $\left\langle p_{y}^{1}\right\rangle_{\mathrm{DI}} /\left\langle p_{y}\right\rangle_{\mathrm{SI}}$ (red dotted line with squares) as a function of the intensity of the laser field.

decrease with increasing intensity, for the intensities considered. Moreover, in figure 6(b), it is shown that it is $\left\langle p_{y}^{1}\right\rangle_{\mathrm{DI}}$ of the tunneling electron that contributes the most to $\left\langle p_{y}^{1}+p_{y}^{2}\right\rangle_{\mathrm{DI}}$ for the intensities considered. The ratio $\left\langle p_{y}^{1}\right\rangle_{\mathrm{DI}} /\left\langle p_{y}\right\rangle_{\mathrm{SI}}$ has surprisingly large values at intensities smaller than the intensities satisfying the criterion for the onset of magnetic field effects $\beta_{0} \approx 1$ a.u. [4, 5]. In contrast, we find that $\left\langle p_{y}\right\rangle_{\text {SI }}$ increases from 0.0035 a.u. at $0.7 \times$ $10^{15} \mathrm{~W} \mathrm{~cm}^{-2}$ to 0.028 a.u. at $4.8 \times 10^{15} \mathrm{~W} \mathrm{~cm}^{-2}$, see figure 6(a) and [20]. The small values of the average electron momentum in SI and the increase of this average with intensity are in accord with the effect of the $\mathbf{F}_{\mathbf{B}}$ force. The latter increases with intensity, since the magnetic field increases. The increase of $\left\langle p_{y}\right\rangle_{\mathrm{SI}}$ with intensity has been addressed in several experimental and theoretical studies [14-18].

In what follows, we show that non-dipole recollisiongated ionization accounts for the large values of $\left\langle p_{y}^{1}\right\rangle_{\mathrm{DI}} /\left\langle p_{y}\right\rangle_{\mathrm{SI}}$ and thus for the large average sum of the two-electron momenta along the propagation direction of the laser field at smaller intensities of $(1.3)-(2) \times 10^{15} \mathrm{~W} \mathrm{~cm}^{-2}$. To do so, we express $\left\langle p_{y}^{i}\right\rangle_{\mathrm{DI}}$ as

$$
\left\langle p_{y}^{i}\right\rangle_{\mathrm{DI}}=\int_{0 \circ}^{180 \circ}\left\langle p_{y}^{i}(\phi)\right\rangle_{\mathrm{DI}} P_{i}^{\mathrm{DI}}(\phi) \mathrm{d} \phi,
$$

with $i=1,2$ for electrons 1 and 2 in double ionization. A similar expression holds in SI. We have already shown that non-dipole recollision-gated ionization accounts for the asymmetry in $P_{1}^{\mathrm{DI}}(\phi)$. Next, we investigate the influence of the magnetic field on $\left\langle p_{y}^{i}(\phi)\right\rangle_{\mathrm{DI}}$. To do so, $\left\langle p_{y}^{1}(\phi)\right\rangle_{\mathrm{DI}}$ of the tunnel electron, $\left\langle p_{y}^{2}(\phi)\right\rangle_{\mathrm{DI}}$ of the bound electron and $\left\langle p_{y}(\phi)\right\rangle_{\mathrm{SI}}$ are plotted in figures 7 (a)-(c), respectively, at $2 \times 10^{15}$ $\mathrm{W} \mathrm{cm}{ }^{-2}$ with linearly polarized light and with the magnetic field switched-on and off. It is shown that the magnitude of the average electron momentum increases as a function of $\phi$,
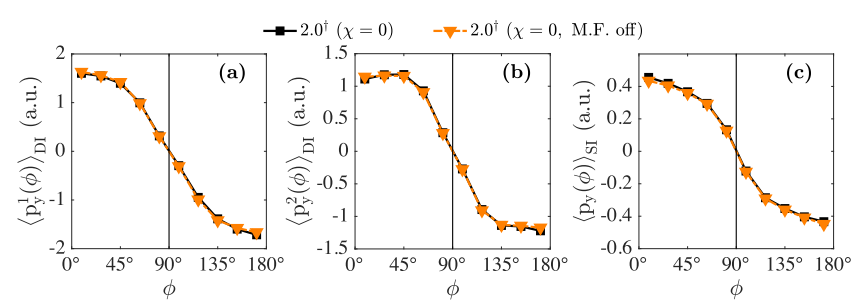

Figure 7. (a) $\left\langle p_{y}^{1}\right\rangle_{\text {DI }}$ of the tunneling electron, (b) $\left\langle p_{y}^{2}\right\rangle_{\text {DI }}$ of the initially bound electron and (c) $\left\langle p_{y}\right\rangle_{\mathrm{SI}}$ in single ionization are plotted as a function of $\phi$ at $2 \times 10^{15} \mathrm{~W} \mathrm{~cm}^{-2}$ with the magnetic field switched-on and off. $\phi$ is binned in intervals of $18^{\circ}$. $\dagger$ Denotes a multiplication factor of $10^{15} \mathrm{~W} \mathrm{~cm}^{-2}$.

both in double and in SI. This is evident mostly for $\phi$ around $0^{\circ}$ and $180^{\circ}$. Moreover, it is clearly seen that the magnetic field has no influence on any of the average electron momenta considered and that $\left\langle p_{y}^{i}(\phi)\right\rangle_{\mathrm{DI}}=\left\langle p_{y}^{i}\left(180^{\circ}-\phi\right)\right\rangle_{\mathrm{DI}}$ and $\left\langle p_{y}(\phi)\right\rangle_{\mathrm{SI}}=\left\langle p_{y}\left(180^{\circ}-\phi\right)\right\rangle_{\mathrm{SI}}$. This is expected when the magnetic field is switched-off, since there is no preferred direction of electron escape on the plane that is perpendicular to the polarization direction ( $x$-axis) of the laser field. We find that similar results hold at $1.3 \times 10^{15} \mathrm{~W} \mathrm{~cm}^{-2}$ and at $3.8 \times 10^{15} \mathrm{~W} \mathrm{~cm}^{-2}$.

We also find (not shown) that the effect of the magnetic field on $\left\langle p_{y}^{1}(\phi)\right\rangle_{\mathrm{DI}}$ is very small even at a more differential level. Specifically, the dependence of $\left\langle p_{y}^{1}(\phi, t)\right\rangle_{\mathrm{DI}}$ on time is very similar both with the magnetic field switched-on and off. The only difference is a small oscillation due to the magnetic field. It is noted that $\left\langle p_{y}^{1}(\phi, t \rightarrow \infty)\right\rangle_{\mathrm{DI}}=\left\langle p_{y}^{1}(\phi)\right\rangle_{\mathrm{DI}}$. We find that similar results (not shown) hold for the bound electron.

Using equation (3), we can now explain why $\left\langle p_{y}^{1}\right\rangle_{\text {DI }}$ is much larger than $\left\langle\mathrm{p}_{\mathrm{y}}\right\rangle_{\mathrm{SI}}$ at intensities around $2 \times$ $10^{15} \mathrm{~W} \mathrm{~cm}^{-2}$. When the magnetic field is switched-on, $\left\langle p_{y}^{1}(\phi)\right\rangle_{\mathrm{DI}}$ does not change but $P_{1, \text { asym }}^{\mathrm{DI}}(\phi)$ does. $P_{1, \text { asym }}^{\mathrm{DI}}(\phi)$ is much wider than $P_{\text {asym }}^{\mathrm{SI}}(\phi)$ and than $P_{2 \text {,asym }}^{\mathrm{DI}}(\phi)$ and has higher values at $2 \times 10^{15} \mathrm{~W} \mathrm{~cm}^{-2}$ rather than at $3.8 \times 10^{15} \mathrm{~W} \mathrm{~cm}^{-2}$. The higher values of $P_{1, \text { asym }}^{\mathrm{DI}}(\phi)$ over a wider range of $\phi$ compared to $P_{\text {asym }}^{\mathrm{SI}}(\phi)$ and $P_{2 \text {,asym }}^{\mathrm{DI}}(\phi)$ result in smaller $\phi$ and thus larger $\left\langle p_{y}^{1}(\phi)\right\rangle_{\mathrm{DI}}$ (figure 7 ) having a significant weight in equation (3).

\section{Conclusions}

We account for non-dipole effects in double ionization. We show that the recollision and the magnetic field act together as a gate. This gate gives rise to a distribution of the $\mathrm{y}$-component of the tunneling electron initial momentum, $P\left(p_{y, t_{0}}^{1}\right)$, which is shifted towards negative values; negative values in the $y$-axis are opposite to the propagation direction of the laser field. The term non-dipole recollision-gated ionization was adopted to describe this effect. We show that this asymmetry in $P\left(p_{y, t_{0}}^{1}\right)$ maps in time to an asymmetry of the transverse electron 1 momentum just before the time of recollision, i.e. to an asymmetry in $P\left(p_{y, t_{\mathrm{r}}}^{1}\right)$. Namely, the $\mathrm{y}$-component of the momentum of the tunneling electron is 
shifted towards positive values just before the time of recollision. Moreover, the asymmetry in $\mathrm{P}\left(p_{y, t_{0}}^{1}\right)$ maps in time to an asymmetry of the transverse electron 1 position just before the time of recollision. Namely, the y-component of the position of the tunneling electron is shifted towards negative values just before the time of recollision.

The above asymmetries combined with the tunneling electron escaping at glancing angles following a recollision give rise to an asymmetry in $P_{1}^{\mathrm{DI}}(\phi)$ with respect to $\phi=90^{\circ}$. The latter is the probability distribution of electron 1 to escape with an angle $\phi$. We find the asymmetry in $P_{1}^{\mathrm{DI}}(\phi)$ to be more significant, i.e. higher values of $P_{1}^{\mathrm{DI}}$ asym $(\phi)$ over a wider range of $\phi$, in double ionization compared to SI. Moreover, we find that higher values of $P_{1 \text {, asym }}^{\mathrm{DI}}(\phi)$ over a wider range of $\phi$ result from larger widths of $P\left(p_{y, t_{\mathrm{r}}}^{1}\right)$ just before the time of recollision. The latter width depends on the intensity and the ellipticity of the laser pulse and we show that it is related to Coulomb focusing. We also show that it is the asymmetry in $P_{1}^{\mathrm{DI}}(\phi)$ over a wide range of $\phi$ that accounts for the large values of the average transverse electron 1 momentum and thus of the large average sum of the two-electron momenta at smaller intensities. Even though not as pronounced, we find these features of the probability distribution $P_{1}^{\mathrm{DI}}(\phi)$ of the tunneling electron to also be present in an experimentally accessible observable. Namely, the probability distribution for electron 1 or 2 to escape with an angle $\phi$. This observable effect of the non-dipole recollision-gated ionization can be measured by future experiments.

Finally, in the current work we show that magnetic field effects cause an offset of the transverse momentum and position of the recolliding electron just before recollision takes place. We show how these asymmetries lead to asymmetries in observables experimentally accessible. We conjecture that this is a general phenomenon not restricted to magnetic field effects. Namely, these observable asymmetries will be present in any process that has two delayed steps and allows an electron to gain an offset before recollision takes place.

\section{Acknowledgments}

AE acknowledges the EPSRC grant no. J0171831 and the use of the computational resources of Legion at UCL.

\section{ORCID iDs}

A Emmanouilidou (10 https://orcid.org/0000-0002-6255-9622

\section{References}

[1] Taylor K T, Parker J S, Dundas D and Meharg K J 2007 J. Mod. Opt. 541959

[2] Becker A, Dörner R and Moshammer R 2005 J. Phys. B 38 S753

[3] Milošević D B, Paulus G G, Bauer D and Becker W 2006 J. Phys. B 39 R203

[4] Reiss H R 2008 Phys. Rev. Lett. 101043002

[5] Reiss H R 2014 J. Phys. B 47204006

[6] Palaniyappan S, DiChiara A, Chowdhury E, Falkowski A, Ongadi G, Huskins E L and Walker B C 2005 Phys. Rev. Lett. 94243003

[7] Lötstedt E and Midorikawa K 2013 Phys. Rev. A 87013426

[8] Emelin M Y and Ryabikin M Y 2014 Phys. Rev. A 89013418

[9] Chirilă C C, Kylstra N J, Potvliege R M and Joachain C J 2002 Phys. Rev. A 66063411

[10] Walser M W, Keitel C H, Scrinzi A and Brabec T 2000 Phys. Rev. Lett. 855082

[11] Keitel C H and Knight P L 1995 Phys. Rev. A 511420

[12] Ludwig A, Maurer J, Mayer B W, Phillips C R, Gallmann L and Keller U 2014 Phys. Rev. Lett. 113243001

[13] Wolter B, Pullen M G, Baudisch M, Sclafani M, Hemmer M, Senftleben A, Schröter C D, Ullrich J, Moshammer R and Biegert J 2015 Phys. Rev. X 5021034

[14] Smeenk C T L, Arissian L, Zhou B, Mysyrowicz A, Villeneuve D M, Staudte A and Corkum P B 2011 Phys. Rev. Lett. 106193002

[15] Chelkowski S, Bandrauk A D and Corkum P B 2014 Phys. Rev. Lett. 113263005

[16] Chelkowski S, Bandrauk A D and Corkum P B 2015 Phys. Rev. A 92 051401(R)

[17] Titi A S and Drake G W F 2012 Phys. Rev. A 85 041404(R)

[18] Ivanov I A 2015 Phys. Rev. A 91043410

[19] Reiss H R 2013 Phys. Rev. A 87033421

[20] Emmanouilidou A and Meltzer T 2017 Phys. Rev. A 95 033405

[21] Emmanouilidou A 2008 Phys. Rev. A 78023411

[22] Emmanouilidou A, Parker J S, Moore L R and Taylor K T 2011 New J. Phys. 13043001

[23] Kustaanheimo P and Stiefel E 1965 J. Reine Angew. Math. 218204

[24] Landau L D and Lifshitz E M 1977 Quantum Mechanics (New York: Pergamon)

[25] Delone N B and Krainov V P 1991 J. Opt. Soc. Am. B 81207

[26] Yakaboylu E, Klaiber M, Bauke H, Hatsagortsyan K Z and Keitel C H 2013 Phys. Rev. A 88063421

[27] Abrines R and Percival I C 1966 Proc. Phys. Soc. London $\mathbf{8 8} 861$

[28] Emmanouilidou A and Staudte A 2009 Phys. Rev. A 80 053415

[29] Corkum P B 1993 Phys. Rev. Lett. 711994

[30] Wang P, Sayler A M, Carnes K D, Esry B D and Ben-Itzhak I 2005 Opt. Lett. 30664

[31] Brabec T, Ivanov M Y and Corkum P B 1996 Phys. Rev. A 54 R2551

[32] Comtois D, Zeidler D, Pepin H, Kiefer J C, Villeneuve D M and Corkum P B 2005 J. Phys. B 381923 